\title{
The pathogenesis and available prevention options in patients with diabetic thrombophilia
}

\author{
Jacek Kwiatkowski, ${ }^{1, A-D, F}$, Jowita Halupczok-Żyła 2,B-D,F, \\ Marek Bolanowski ${ }^{2, E, F}$, Małgorzata Kuliszkiewicz-Janus, ${ }^{1, E, F}$ \\ 1 Department and Clinic of Hematology, Blood Neoplasms and Bone Marrow Transplantation, Wroclaw Medical University, Poland \\ ${ }^{2}$ Department and Clinic of Endocrinology, Diabetology and Isotope Therapy, Wroclaw Medical University, Poland \\ A - research concept and design; $B$ - collection and/or assembly of data; $C$ - data analysis and interpretation; \\ $D$ - writing the article; $E$ - critical revision of the article; $F$ - final approval of the article
}

Address for correspondence

Jacek Kwiatkowski

E-mail: j.k.kwiatkowski@wp.pl

Funding sources

None declared

Conflict of interest

None declared

Received on April 23, 2017

Reviewed on April 27, 2017

Accepted on May 5, 2017

\begin{abstract}
Diabetes mellitus (DM), a growing health problem itself, is accompanied by an increased risk of cardiovascular and thrombotic complications. The imbalance between coagulation and fibrinolysis processes observed in patients with diabetes may be defined as diabetic thrombophilia. Several mechanisms are involved in the hypercoagulability state in diabetics, including endothelial cell damage, altered platelet structure and function, increased microparticle formation, different structure of fibrin clots, disturbances in the activity of coagulation factors, fluctuations in the concentrations of fibrinolysis activators and inhibitors, and qualitative changes of proteins due to glycation and oxidation processes. These all are the reasons why DM is the most common cause of acquired thrombophilia. Moreover, diabetes changes the efficacy of certain medications. Results of various trials seem to suggest that thrombolytic drugs are less effective in patients suffering from this disease. The impact of DM on the effectiveness of treatment with acetylsalicylic acid (ASA) remains unclear. Awareness of thrombotic complications in diabetic patients may enable earlier diagnosis and proper therapy.
\end{abstract}

Key words: diabetic thrombophilia, diabetes mellitus, thrombosis, coagulation factors, hypercoagulability

DOI

10.17219/acem/71054

\section{Copyright}

Copyright by Author(s)

This is an article distributed under the terms of the

Creative Commons Attribution Non-Commercial License

(http://creativecommons.org/licenses/by-nc-nd/4.0/) 


\section{Introduction}

Thrombophilia (hypercoagulability) is a congenital or acquired condition caused by the predominance of coagulation processes over fibrinolysis and resulting in thrombosis. The most common congenital causes are factor $\mathrm{V}$ Leiden mutation, prothrombin G20210A mutation, protein $S$ deficiency, protein $\mathrm{C}$ deficiency, antithrombin deficiency, and MTHFR gene mutations. Acquired thrombophilia can be caused by antiphospholipid syndrome or drugs, such as oral and transdermal contraceptives or chemotherapeutic agents. ${ }^{1}$

Diabetes is a group of metabolic diseases characterized by high blood sugar levels over a long period. It is associated with an increased risk of thrombotic and cardiovascular complications, such as myocardial infarction and ischemic stroke, which are the most common causes of death among diabetics. ${ }^{2}$

The imbalance between the clotting system coagulation and fibrinolysis (favoring coagulation) in patients with diabetes is described as diabetic thrombophilia. ${ }^{3}$ Diabetes mellitus (DM) is considered to be a leading cause of acquired thrombophilia. ${ }^{4}$ The aim of our paper was to investigate the pathogenesis of diabetic thrombophilia, and to discuss primary and secondary prevention strategies.

\section{Endothelial dysfunction}

Endothelium is the lining in all the blood vessels of the human body and it plays an important role in the regulation of the hemostatic processes. ${ }^{5}$ In physiologic conditions, due to its antithrombotic activity, the endothelium maintains continuous blood flow through the inhibition of platelet aggregation and of the inflammatory activation of leukocytes and coagulation factors. Endothelial function is already impaired in patients with insulin resistance, diabetes or in individuals at a high risk for developing type 2 diabetes (T2D), which is further worsened by hypertension, dyslipidemia and nicotinism. ${ }^{6,7}$ The biochemical and cellular factors behind endothelial dysfunction in diabetes include increased activation of protein kinase $C$, elevated levels of growth factors (endothelin, angiotensin II and/or cytokines), nonenzymatic glycation of proteins and other molecules, oxidative stress, and impaired insulin activation of phosphatidylinositol $(3,4,5)$-triphosphate (PIP-3) kinase, but normal response of mitogen-activated protein kinase (MAP). ${ }^{7}$ It is worth mentioning that in T2D, endothelial progenitor cells (EPCs) count is low and their functionality is impaired. Endothelial progenitor cells are responsible for blood vessel formation and they support the repair of damaged endothelium. Although the mechanism of EPCs dysfunction is not fully understood, it is suggested that hyperglycemia-induced increased oxidative stress is an important factor in this process. ${ }^{8}$

The well-known mechanisms contributing to endothelial dysfunction phenotype in T2D are impaired glucose metabolism, altered insulin signaling and low-grade inflammatory state. ${ }^{9}$ Many important relationships between insulin resistance and endothelial dysfunction have been discussed in the literature. In insulin resistance, the phosphatidylinositol 3-kinase-dependent signaling is altered, causing an imbalance between the production of nitric oxide (NO) and the secretion of endothelin-1. This leads to a decrease in blood flow, which exacerbates insulin resistance. Besides the regulation of endothelial NO and endothelin-1 synthesis, insulin also stimulates the expression of vascular cell adhesion molecule (VCAM)-1 and E-selectin on endothelium. ${ }^{10}$ Moreover, by acting through monocyte receptors, insulin may produce anti-inflammatory effects. ${ }^{11}$

The consequences of endothelial dysfunction include increased vascular permeability, decrease in heparin sulphate concentration, reduced production of $\mathrm{NO}$, reduced release of microparticles, and the expression of adhesion molecules, which allow leukocyte migration during inflammation. Damage of endothelial cells leads to the exposition of subendothelial matrix, which has prothrombic properties. ${ }^{12}$ In recent years, much attention has been paid to the procoagulant activity of microparticles, which are small fragments of cell membrane vesicles, released mainly from the platelets. They play a significant role in diabetic atherogenesis as a reservoir of bioactive substances involved in inflammatory and thrombotic processes. The concentration of microparticles is dependent on the degree of glycemic control and in the future it may become a clinically relevant biomarker of diabetic vascular dysfunction. ${ }^{13}$

Additionally, hyperhomocysteinemia and hyperuricemia, through their direct cytotoxic effect on endothelium, promote thrombosis and formation of atherosclerotic lesions in obese patients. ${ }^{14}$ The degree of endothelial cell injury is correlated with increased concentrations of interleukin 6 (IL-6), tumor necrosis factor alpha (TNF- $\alpha$ ) and C-reactive protein (CRP). ${ }^{15}$ Hemostasis and inflammation are closely interrelated processes, responsible for the restoration of normal tissue function after injury. The cooperation between the immune and hemostatic systems takes place on multiple levels and involves many hemostatic components, such as vascular EPCs, platelets, plasma coagulation cascade, anticoagulant pathways, and fibrinolytic activity. The main proinflammatory cytokines linked to the activation of thrombotic process are TNF- $\alpha$, IL-1 and IL- $6 .{ }^{16}$ A recent report presented a novel inflammatory pathway, in which Wnt5a signaling and c-Jun $\mathrm{N}$-terminal kinase (JNK) activation mediate impaired endothelial function in diabetes. ${ }^{17}$

It should be noted that endothelial dysfunction may precede the development of T2D. Meigs et al. showed that plasma markers of endothelial dysfunction (plasminogen activator inhibitor-1 antigen and von Willebrand factor antigen) increased the risk of incident diabetes independently of other diabetes risk factors such as obesity, insulin resistance and inflammation. ${ }^{18}$ In a population-based study, biomarkers of oxidative stress, inflammation and 
endothelial dysfunction predicted type 2 diabetes. ${ }^{19}$ Biomarkers of oxidative stress, F2-isoprostanes and oxidized low-density lipoprotein (LDL) were positively associated with incident T2D; however, the associations were attenuated by the adjustment for body mass index (BMI). ${ }^{20}$

\section{Altered platelet function in patients with diabetes}

Platelet involvement in the pathogenesis and development of diabetic macro- and microangiopathy has been proven in many studies. ${ }^{21,22}$ Platelets, due to their ability to interact with endothelial cells, are the key elements of thrombotic and embolic complications. Recently, many disturbances concerning platelet structure and function in patients with diabetes have been described. They include increased platelet volume, altered plasma membrane fluidity, elevated concentration of cell calcium, increased $\mathrm{P}$-selectin expression, reduced production of $\mathrm{NO}$, diminished sensitivity to antiaggregating agents (such as $\mathrm{NO}$ and prostacyclin - PGI 2), and enhanced activation of the arachidonic acid pathway, resulting in increased thromboxane $\mathrm{A}_{2}$ synthesis. ${ }^{23-25}$ Another finding concerning the function of platelets in diabetics was that they produce more tissue factor (TF) than platelets in control subjects due to the loss of insulin inhibition. ${ }^{26}$ These changes cause firm adhesion of platelets and their aggregation, as well as their interaction with monocytes, granulocytes, erythrocytes, and endothelial cells. ${ }^{27}$ Active platelets are also the source of aforementioned microparticles. ${ }^{28}$

Soma et al. showed that platelet surface markers and the percentage of activated platelets were elevated in diabetics with and without cardiovascular manifestation compared to healthy individuals. The highest percentage of activated platelets and increased microparticle formation were observed in the group of patients with diabetes and cardiovascular complications. ${ }^{29}$

Increased platelet reactivity in diabetics is caused by many factors, e.g., hyperglycemia, hypertriglyceridemia, oxidative stress, inflammation, and absolute or relative insulin deficiency, which further favor the development of vascular complications. ${ }^{30}$ Hyperglycemia following disturbances in insulin secretion, insulin action or both may be a cause of platelet hyperactivity in subjects with diabetes. It is established that hyperglycemia disrupts $\mathrm{Ca}^{2+}$ homeostasis in platelets by mobilizing $\mathrm{Ca}^{2+}$ from intracellular storage pools, which leads to an increase in intracellular $\mathrm{Ca}^{2+}$ levels. Moreover, hyperglycemia may promote nonenzymatic glycation of platelet membrane proteins with changes in protein structure and conformation, as well as alterations of membrane lipid dynamics.

The other mechanism is impaired platelet response to high insulin levels (platelet insulin resistance), associated with a change in intracellular signal transduction and the suppression of the action of inhibiting factors (fibrin degradation products - FDP). ${ }^{31}$ Insulin regulates platelet function by cell surface receptors, counteracting the activation and aggregation of platelets. It also sensitizes platelets to PGI 2 and NO actions, inhibiting their aggregation, and reduces the prothrombotic effects of a number of agonists (adenosine diphosphate (ADP), collagen, thrombin, and epinephrine). ${ }^{30}$ It has been shown that insulin maintains platelet sensitivity to PGI 2 by increasing surface expression of PGI 2 receptors. ${ }^{9}$ Moreover, insulin increases platelet concentrations of both cyclic adenosine monophosphate (cAMP) and cyclic guanosine monophosphate (cGMP), which are the main inhibitory second messengers for platelet activation. Another study, conducted by Ferreira et al., revealed that insulin attenuates platelet functions by interfering with cAMP suppression through insulin receptor substrate-1 (IRS-1) and G-protein (Gi). The inhibition of Gi activity by insulin is associated with a decrease in $\mathrm{Ca}^{2+}$ mobilization and the reduction of adhesion and aggregation. Thus, the authors speculated that the hyperresponsiveness of platelets in diabetes explains the absence of platelet inhibition by insulin. ${ }^{25}$ It has been shown that platelet hyperactivity in T2D is probably caused by a defect in the mechanisms through which insulin interferes with signaling by the P2Y12 receptor. ${ }^{32}$ The other mentioned cause of high platelet activity is decreased number and affinity of platelet insulin receptor (IR). ${ }^{9}$ In addition, Zhang et al. demonstrated that genetic background might play a role in diminished antiplatelet efficacy of dual antiplatelet therapy. The authors presented an association between IRS-1 polymorphisms and high platelet reactivity in subjects with $\mathrm{T} 2 \mathrm{D}$ and coronary artery disease (CAD). ${ }^{32}$

Several biomarkers of diabetic thrombocytopathy have been proposed to be used in clinical practice. Since platelet volume indices (PVI), including mean platelet volume (MPV), platelet distribution width (PDW) and plateletlarge cell ratio (P-LCR) are considered indicators of platelet hyperactivity, they can be utilized as potential biomarkers for diabetic complications. Recently, Buch et al. determined MPV and PDW as predictive biomarkers of diabetic vascular complications (their prognostic value is more significant for microvascular than macrovascular complications). Moreover, it was found that improved glycemic control decreases MPV, which indicates that diminished platelet activity achieved by proper glycemic control may delay or even prevent vascular complications. In a cross-sectional study, a group of patients with glycated hemoglobin (HbA1c) $>7.0 \%$ had higher MPV than a group with $\mathrm{HbA} 1 \mathrm{c} \leq 7.0 \%$. The authors found a positive correlation between MPV and both short-term and long-term glycemic markers in subjects with T2D. It has been hypothesized that an increase in MPV can result from hyperglycemia, which exerts direct osmotic effects on platelets, causing osmotic swelling. In addition, increased MPV may be associated with a higher platelet turnover rate. ${ }^{4,33}$ 


\section{Coagulation factors and fibrinolysis}

Changes in the concentration and activity of coagulation factors in patients with diabetes lead to the dominance of thrombogenesis over fibrinolysis. The common feature of this phenomenon is the influence of chronic hyperglycemia on the increase of TF expression and the activity of factor VII (responsible for the activation of coagulation in the extrinsic pathway), II, V, VIII, and X. Moreover, the concentration of $\mathrm{C}$ - and $\mathrm{S}$-protein (coagulation cascade inhibitors) is decreased. The changes mentioned above contribute to hypercoagulability by enhanced thrombin generation. ${ }^{34,35}$ Von Willebrand factor (vWF) mediates the protection of factor VIII from proteolysis and is prerequisite for platelets adhesion to subepithelium. ${ }^{36}$ Its elevated plasma levels have been observed in patients with poor-controlled DM and with cardiovascular disease. ${ }^{37}$ Tissue factor pathway inhibitor (TFPI) concentration is conversely correlated to fasting glucose and HbAlc. ${ }^{38,39}$ An essential reaction in the fibrinolysis process is the conversion of plasminogen to plasmin, which causes the proteolysis of fibrin clots. This reaction is controlled by activators (tissue plasminogen activators - t-PA) and inhibitors $(\alpha 2$-antiplasmin, $\alpha 2$-macroglobulin, plasminogen activator inhibitor - PAI). ${ }^{40}$ Apart from quantitative changes in coagulation factors and fibrinolysis, in chronic hyperglycemia there are also qualitative changes due to glycation and oxidation processes. Protein glycation is covalent bonding (post translational modification) of a simple sugar molecule to free amino groups of proteins, without the controlling action of an enzyme. The amino groups of lysine in fibrinogen could be bonded with the carbonyl groups of glucose. Hyperglycemia increases plasma protein glycation. The absence of free amino groups of lysine, which are a binding site for fibrinolytic proteins ( $\mathrm{t}-\mathrm{PA}$ and plasminogen) to fibrin, impairs clot lysis. Additionally, the glycation of plasminogen decreases the specific activity of plasmin and reduces its profibrinolytic properties. ${ }^{41}$ There is a noticeable difference in the structure of fibrin clots, which are more compact and, therefore, less susceptible to fibrinolysis. The observed increased fibrinogen levels, an independent risk factor for atherosclerosis and cardiovascular disease, may be a predisposing factor to the deposition of fibrin clots (a potential role in the development of diabetic nephropathy) and increased platelet aggregation. ${ }^{42}$ Hess et al. demonstrated that incorporating complement component 3 into the fibrin clots of patients suffering from diabetes had a greater effect on the prolongation of clot lysis compared to the clots from healthy controls. ${ }^{43}$ Fibrinolysis impairment in diabetic patients depends primarily on the increased concentration of PAI, which is embedded in fibrin clots by activated factor XIII. Moreover, Hori et al. noticed that diabetes patients had an elevated level of thrombin activatable fibrinolysis inhibitor (TAFI), which protects fibrin clots against lysis. ${ }^{44}$

\section{Treatment and prophylaxis}

Both prophylaxis and treatment of thrombophilia associated with diabetes should be based primarily on the well-controlled disease, close to normoglycemia, measured by glycated hemoglobin. ${ }^{45}$ It is noticeable that hypoglycemia can also lead to hypercoagulability due to elevated fibrinogen and PAI levels. ${ }^{46}$ Another very important issue concerns the prevention of atherosclerosis, which can be achieved by a change of dietary habits, enhancement of physical activity (up to moderate-intensity exercise for $30 \mathrm{~min}, 5$ times a week) and smoking cessation. Decreased concentration of LDL, stabilization of atherosclerotic plaque and anti-inflammatory effect are benefits of therapy with 3-hydroxy-3-methyl-glutaryl-coenzyme A (HMG-CoA) reductase inhibitors (statins). According to the observations of Kim et al., diabetic patients treated with statins or angiotensin receptor blockers showed a decreased endogenous thrombin potential ratio and increased protein $\mathrm{C}$ levels. ${ }^{34}$ Because of a high risk of myocardial infarction and ischemic stroke, it is advisable to use acetylsalicylic acid (ASA) at a prophylactic dose. However, diabetic patients have a high prevalence of suboptimal effect or even aspirin resistance. ${ }^{47}$ Depending on the cause, various groups of drugs are being used to treat the hypercoagulation complications of diabetic thrombophilia.

Antiplatelet drugs decrease platelet aggregation and inhibit thrombus formation. They are effective in the treatment and prophylaxis of arterial thrombosis. The class of antiplatelet drugs includes, among others, irreversible cyclooxygenase inhibitors (ASA), adenosine diphosphate receptor inhibitors (clopidogrel, prasugrel) and glycoprotein IIB/IIIA inhibitors (abciximab, tirofiban). Bhatt et al. noticed that clopidogrel is superior to ASA in reducing recurrent ischemic events in patients with diabetes, thereby causing fewer bleeding complications. ${ }^{48}$ According to Wiviott et al., oral antiplatelet therapy provided with prasugrel is of particular benefit to patients with diabetes. They compared prasugrel to clopidogrel in the secondary prevention of major cardiovascular events. ${ }^{49}$ In the observation by Perkan et al., abciximab treatment was associated with a lower in-hospital rate and 30-day mortality rate, and a lower incidence of death and reinfarction at 30 days in diabetic patients undergoing primary percutaneous coronary intervention due to acute myocardial infarction..$^{50}$

Anticoagulants inhibit the coagulation cascade that happens after the initial platelet aggregation. They are used in the treatment and prophylaxis of venous thrombosis. Anticoagulants are divided into: vitamin $\mathrm{K}$ antagonists (warfarin, acenocoumarol), direct factor Xa inhibitors (rivaroxaban, apixaban), direct thrombin inhibitors (bivalirudin, dabigatran), and unfractionated heparin (UFH), as well as low-molecular-weight heparin (enoxaparin, nadroparin, dalteparin). Bansilal et al. aimed to examine the safety and efficacy of rivaroxaban vs warfarin in patients with non-valvular atrial fibrillation and diabetes. The relative 
efficacy and safety of both drugs was similar. ${ }^{51}$ According to the observations of Morrow et al., in high-risk ST elevation myocardial infarction (STEMI) patients with diabetes undergoing fibrinolysis, a reperfusion strategy with the use of enoxaparin significantly improved the outcomes compared to UFH. ${ }^{52}$

The last group of drugs includes thrombolytic drugs, which are responsible for dissolving blood clots. These include streptokinase, urokinase and recombinant t-PA (alteplase, reteplase). Masoomi et al. noticed that complete ST-resolution after streptokinase infusion occurred in $31.6 \%$ of diabetic and $51.0 \%$ of non-diabetic patients, respectively. ${ }^{53}$ The failure of ST-segment resolution $180 \mathrm{~min}$ after drug application was notably higher in diabetic than nondiabetic patients. Another observation by Strbian et al. was that poor outcome of thrombolysis after ischemic stroke patients was associated with diabetes and elevated admission blood glucose. ${ }^{54}$ The results of trials seem to suggest that thrombolytic drugs are less effective in the case of patients suffering from diabetes.

\section{Conclusions}

The pathogenesis of diabetic thrombophilia is not fully understood. Despite a number of studies available on this issue, potential factors and mechanisms that may exacerbate hypercoagulability in diabetic patients are constantly being sought. A better understanding of the causes of hemostatic disorders in these patients makes it possible to provide adequate primary prevention and provides an opportunity to increase the effectiveness of sufficient therapy. Because of the large and still growing population of diabetics, diabetic thrombophilia is a crucial issue because of its complications, such as cardiovascular disease.

\section{References}

1. Mannucci PM, Tripodi A, Bottasso B, et al. Markers of procoagulant imbalance in patients with inherited thrombophilic syndromes. Thromb Haemost. 1992;67(2):200-202.

2. Grant PJ. Diabetes mellitus as a prothrombotic condition. J Intern Med. 2007;262(2):157-172.

3. Kinalska I, Telejko B. Recent progress in diabetic thrombophilia prophylaxis and treatment [in Polish]. Diabetologia Praktyczna. 2003;4(2)153-159.

4. Kadić D, Hasić S, Spahić E. Mean platelet volume predicts the glycemic control deterioration in diabetes mellitus type 2 patients. Med Glas (Zenica). 2016;13(1):1-7.

5. Pasqualini L, Marchesi S, Vaudo G, et al. Association between endothelial dysfunction and major cardiovascular events in peripheral vascular disease. Vasa. 2003;32(3):139-143.

6. Taylor A. Pathophysiology of hypertension and endothelial dysfunction in patients with diabetes mellitus. Endocrinol Metab Clin North Am. 2001;30:983-997.

7. Calles-Escandon J, Cipolla M. Diabetes and endothelial dysfunction: A clinical perspective. Endocr Rev. 2001;22(1):36-52.

8. Hamed S, Brenner B, Roguin A. Nitric oxide: A key factor behind the dysfunctionality of endothelial progenitor cells in diabetes mellitus type-2. Cardiovasc Res. 2011;91(1):9-15.

9. Vazzana N, Ranalli P, Cuccurullo C, Davi G. Diabetes mellitus and thrombosis. Thromb Res. 2012;129(3):371-377.
10. Kim J, Montagnani M, Kon Koh K, Quon MJ. Reciprocal relationships between insulin resistance and endothelial dysfunction: Molecular and pathophysiological mechanisms. Circulation. 2006;113:1888-1904.

11. Vinik AJ, Erbas T, Park TS, Nolan R, Pittenger GL. Platelet dysfunction in type 2 diabetes. Diabetes Care. 2001;24(8):1476-1485.

12. Alexandru N, Badila E, Weiss E, Cochior D, Stępień E, Georgescu A. Vascular complications in diabetes: Microparticles and microparticle-associated microRNAs as active players. Biochem Biophys Res Commun. 2016;472(1):1-10.

13. Kundi $\mathrm{H}$, Kiziltunc $\mathrm{E}$, Ates $\mathrm{I}$, et al. Association between plasma homocysteine levels and end-organ damage in newly diagnosed type 2 diabetes mellitus patients. Endocr Res. 2015;25:1-6.

14. Akbas EM, Timuroglu A, Ozcicek A, et al. Association of uric acid, atherogenic index of plasma and albuminuria in diabetes mellitus. Int J Clin Exp Med. 2014;7(12):5737-5743.

15. Yudkin J, Stehouwer CD, Emeis JJ, Coppack SW. C-reactive protein in healthy subjects. Associations with obesity, insulin resistance, and endothelial dysfunction: A potential role for cytokines originating from adipose tissue? Arterioscler Thromb Vasc Biol. 1999;19:972-978.

16. Margetic S. Inflammation and haemostasis. Biochem Med (Zagreb). 2012;22(1):49-62.

17. Bretón-Romero R, Feng B, Holbrook M, et al. Endothelial dysfunction in human diabetes is mediated by Wnt5a-JNK signaling. Arterioscler Thromb Vasc Biol. 2016;36(3):561-569.

18. Meigs JB, O'Donnell CJ, Tofler GH, et al. Hemostatic markers of endothelial dysfunction and risk of incident type 2 diabetes: The Framingham Offspring Study. Diabetes. 2006;55(2):530-537.

19. Leurs PB, Stolk RP, Hamulyak K, Van Oerle R, Grobbee DE, Wolffenbuttel $\mathrm{BH}$. Tissue factor pathway inhibitor and other endotheliumdependent hemostatic factors in elderly individuals with normal or impaired glucose tolerance and type 2 diabetes. Diabetes Care. 2002;25(8):1340-1345.

20. Odegaard AO, Jacobs DR, Sanchez OA, GoffDC Jr, Reiner AP, Gross MD. Oxidative stress, inflammation, endothelial dysfunction and incidence of type 2 diabetes. Cardiovasc Diabetol. 2016;15:51.

21. Sobol AB, Watala $C$. The role of platelets in diabetes-related vascular complications. Diabetes Res Clin Pract. 2000;50(1):1-16.

22. Dinda AK, Kumar R, Saraya AK. Platelet hyperreactivity and vasculopathy in insulin dependent diabetes mellitus. Platelets. 1992;3(2):83-86.

23. Trovati $M$, Anfossi $G$. Insulin, insulin resistance and platelet function: Similarities with insulin effect on cultured vascular smooth muscle cells. Diabetologia. 1998;41:609-622.

24. Caimi G, Lo Presti R, Montana M, et al. Membrane fluidity, membrane lipid pattern, and cytosolic $\mathrm{Ca}^{2+}$ content in platelets from a group of type II diabetic patients with macrovascular complications. Diabetes Care. 1995;18(1):60-63.

25. Ferreira IA, Eybrechts KL, Mocking Al, Kroner C, Akkerman JW. IRS-1 mediates inhibition of $\mathrm{Ca}^{2+}$ mobilization by insulin via the inhibitory G-protein Gi. J Biol Chem. 2004;279(5):3254-3264.

26. Gerrits AJ, Koekman CA, van Haeften TW, Akkerman JWN. Platelet tissue factor synthesis in type 2 diabetic patients is resistant to inhibition by insulin. Diabetes. 2010;59(6):1487-1495.

27. Tschoepe D, Rauch U, Schwippert B. Platelet-leukocyte cross-talk in diabetes mellitus. Horm Metab Res.1997;29:631-635.

28. Santilli F, Simeone P, Liani R, Davi G. Platelets and diabetes mellitus. Prostaglandins Other Lipid Mediat. 2015;120:28-39.

29. Soma P, Swanepoel AC, du Plooy JN, Mqoco T, Pretorius E. Flow cytometric analysis of platelets type 2 diabetes mellitus reveals "angry" platelets. Cardiovasc Diabetol. 2016;15:52.

30. Lundström A, Laska AC, Von Arbin M, Jörneskog G, Wallén H. Glucose intolerance and insulin resistance as predictors of low platelet response to clopidogrel in patients with minor ischemic stroke or TIA. Platelets. 2014; 25(2):102-110.

31. Wilson P. Effect of fibrinogen degradation products on platelet aggregation. J Clin Pathol. 1968;21(2):147-153.

32. Zhang D, Zhang X, Liu D, et al. Association between insulin receptor substrate-1 polymorphisms and high platelet reactivity with clopidogrel therapy in coronary artery disease patients with type 2 diabetes mellitus. Cardiovasc Diabetol. 2016;15:50

33. Buch A, Kaur S, Nair R, Jain A. Platelet volume indices as predictive biomarkers for diabetic complications in type 2 diabetic patients. J Lab Physicians. 2017;9(2):84-88 
34. Kim HK, Kim JE, Park SH, Kim YI, Nam-Goong IS, Kim ES. High coagulation factor levels and low protein $C$ levels contribute to enhanced thrombin generation in patients with diabetes who do not have macrovascular complications. J Diabetes Complications. 2014;28(3):365-369.

35. Tripodi A, Branchi A, Chantarangkul V, et al. Hypercoagulability in patients with type 2 diabetes mellitus detected by a thrombin generation assay. J Thromb Thrombolysis. 2011;31(2):165-172.

36. Hess K. The vulnerable blood. Coagulation and clot structure in diabetes mellitus. Hamostaseologie. 2015;35(1):25-33.

37. Hernestål-Boman J, Norberg M, Jansson JH, et al. Signs of dysregulated fibrinolysis precede the development of type 2 diabetes mellitus in a population-based study. Cardiovasc Diabetol. 2012;11:152.

38. Ruszkowska-Ciastek B, Sokup A, Wernik T, et al. Low-grade risk of hypercoagulable state in patients suffering from diabetes mellitus type 2. J Zhejiang Univ Sci B. 2015;16(9):788-795.

39. Bratseth V, Byrkjeland R, Njerve IU, Solheim S, Arnesen H, Seljeflot I. Procoagulant activity in patients with combined type 2 diabetes and coronary artery disease: No effects of long-term exercise training. Diab Vasc Dis Res. 2017;14(2):144-151.

40. Lijnen HR, Collen D. Interaction of plasminogen activators and inhibitors with plasminogen and fibrin. Semin Thromb Hemost. 1982;8(1): 2-10.

41. Svensson J, Bergman AC, Adamson U, Blombäck $M$, Wallén $H$, Jörneskog G. Acetylation and glycation of fibrinogen in vitro occur at specific lysine residues in a concentration dependent manner: A mass spectrometric and isotope labeling study. Biochem Biophys Res Commun. 2012;421(2):335-342.

42. Alzahrani $\mathrm{SH}, \mathrm{Ajjan}$ RA. Coagulation and fibrinolysis in diabetes. Diab Vasc Dis Res. 2010;7(4):260-273.

43. Hess K, Alzahrani SH, Mathai M, et al. A novel mechanism for hypofibrinolysis in diabetes: The role of complement C3. Diabetologia. 2012:55(4):1103-1113.

44. Hori Y, Gabazza EC, Yano Y, et al. Insulin resistance is associated with increased circulating level of thrombin-activatable fibrinolysis inhibitor in type 2 diabetic patients. J Clin Endocrinol Metab. 2002;87(2): 660-665.

45. Bell EJ, Selvin E, Lutsey PL, Nambi V, Cushman M, Folsom AR. Glycemia (hemoglobin A1c) and incident venous thromboembolism in the Atherosclerosis Risk in Communities cohort study. Vasc Med. 2013;18(5):245-250.
46. Gogitidze Joy N, Hedrington MS, Briscoe VJ, Tate DB, Ertl AC, Davis SN. Effects of acute hypoglycemia on inflammatory and pro-atherothrombotic biomarkers in individuals with type 1 diabetes and healthy individuals. Diabetes Care. 2010;33(7):1529-1535.

47. Jung JH, Tantry US, Gurbel PA, Jeong Y-H. Current antiplatelet treatment strategy in patients with diabetes mellitus. Diabetes Metab J. 2015; 39(2):95-113.

48. Bhatt DL, Marso SP, Hirsch AT, Ringleb PA, Hacke W, Topol EJ. Amplified benefit of clopidogrel versus aspirin in patients with diabetes mellitus. Am J Cardiol. 2002;90(6):625-628.

49. Wiviott SD, Braunwald E, Angiolillo DJ, et al. Greater clinical benefit of more intensive oral antiplatelet therapy with prasugrel in patients with diabetes mellitus in the trial to assess improvement in therapeutic outcomes by optimizing platelet inhibition with prasugrelthrombolysis in myocardial infarction 38. Circulation. 2008;118(16): 1626-1636.

50. Perkan A, Vitrella G, Barbati G, et al. Impact of abciximab on prognosis in diabetic patients undergoing primary percutaneous coronary intervention. J Cardiovasc Med (Hagerstown). 2013;14(2):127-135.

51. Bansilal S, Bloomgarden Z, Halperin JL, et al.; ROCKET AF Steering Committee and Investigators. Efficacy and safety of rivaroxaban in patients with diabetes and nonvalvular atrial fibrillation: The Rivaroxaban Once-daily, Oral, Direct Factor Xa Inhibition Compared with Vitamin K Antagonism for Prevention of Stroke and Embolism Trial in Atrial Fibrillation (ROCKET AF Trial). Am Heart J. 2015;170(4):675-682.

52. Morrow DA, Antman EM, Murphy SA, et al.; TIMI Study Group. Effect of enoxaparin versus unfractionated heparin in diabetic patients with ST-elevation myocardial infarction in the Enoxaparin and Thrombolysis Reperfusion for Acute Myocardial Infarction Treatment-Thrombolysis In Myocardial Infarction study 25 (ExTRACT-TIMI 25) trial. Am Heart J. 2007; 154(6):1078-1084.

53. Masoomi M, Samadi S, Sheikhvatan M. Thrombolytic effect of streptokinase infusion assessed by ST-segment resolution between diabetic and non-diabetic myocardial infarction patients. Cardiol J. 2012; 19(2):168-173.

54. Strbian D, Piironen K, Meretoja A, et al.; Helsinki Stroke Thrombolysis Registry Group. Intravenous thrombolysis for acute ischemic stroke patients presenting with mild symptoms. Int J Stroke. 2013;8(5):293-299. 